\title{
Time-resolved photoluminescence study of the stimulated emission in $\mathrm{ZnO}$ nanoneedles
}

\author{
W. M. Kwok \\ Department of Chemistry, The University of Hong Kong, Pokfulam Road, Hong Kong \\ A. B. Djurišića) and Y. H. Leung \\ Department of Physics, The University of Hong Kong, Pokfulam Road, Hong Kong \\ W. K. Chan and D. L. Phillips \\ Department of Chemistry, The University of Hong Kong, Pokfulam Road, Hong Kong
}

(Received 24 March 2005; accepted 12 July 2005; published online 23 August 2005)

\begin{abstract}
$\mathrm{ZnO}$ nanoneedles were fabricated by thermal evaporation of $\mathrm{Zn}$ nanoparticles at $800{ }^{\circ} \mathrm{C}$ and atmospheric pressure. The samples showed strong ultraviolet photoluminescence and weak orange defect luminescence. Time-resolved photoluminescence (TRPL) was measured using the Kerr-gated fluorescence technique in order to probe the ultrafast carrier dynamics in exciton-exciton scattering and electron hole plasma (EHP) regimes. In both regimes, the decay time of the photoluminescence is very fast $(\sim 1 \mathrm{ps})$. Even though no structure is detected in the time-integrated spectra of the EHP emission, the TRPL reveals the coexistence of the excitons and free carriers. Possible reasons for the observed phenomena are discussed. (C) 2005 American Institute of Physics.
\end{abstract}

[DOI: $10.1063 / 1.2035871]$

$\mathrm{ZnO}$ nanostructures are of great interest for a wide range of photonic and electronic applications, such as field emission ${ }^{1-3}$ and ultraviolet (UV) lasing. ${ }^{4-16}$ Both field emission and stimulated emission were demonstrated for a variety of morphologies. It is well known that $\mathrm{ZnO}$ nanostructures can exhibit a very wide range of possible morphologies, such as nanowires, ${ }^{1,7,8}$ tetrapods, ${ }^{2,12,16}$ nanoneedles, ${ }^{3}$ nanoribbons, ${ }^{7}$ nanohelixes, ${ }^{4}$ nanorings, ${ }^{4}$ dendritic nanowire arrays, ${ }^{5}$ etc. The field emission properties of $\mathrm{ZnO}$ nanoneedles have been reported, but lasing in these structures has not been studied. While the majority of the time-resolved studies in $\mathrm{ZnO}$ have been performed on nanocrystalline thinfilm samples, ${ }^{6,9-11,13,14}$ time-resolved studies of the stimulated emission in $\mathrm{ZnO}$ nanostructures are scarce. ${ }^{7,8,12}$ In this work, we report room-temperature photoluminescence (PL) and the time-resolved study of the stimulated emission of $\mathrm{ZnO}$ nanoneedles in exciton-exciton (E-E) scattering and electron-hole plasma (EHP) regimes. Time-resolved measurements indicate the coexistence of free carriers and excitons and very short decay times in both emission regimes.

The $\mathrm{ZnO}$ nanoneedles were synthesized by thermally evaporating Zn nanoparticles (99.9\% purity, average particle size $35 \mathrm{~nm}$, obtained from Nanostructured and Amorphous Materials Inc.) under a humidified argon gas flow of 0.25 $\mathrm{lpm}$ at $800{ }^{\circ} \mathrm{C}$ in a horizontal tube furnace. The nanoparticles were placed in a crucible which was then inserted into a tube furnace, which was preheated to the desired temperature. One end of the tube was exposed to the ambient atmosphere. The whole process took place at atmospheric pressure and no catalyst was added. After $30 \mathrm{~min}$ of evaporation, the white/ grey products were deposited on silicon substrates at 725$750^{\circ} \mathrm{C}$. The synthesis procedure is considerably simpler compared to the previous report on $\mathrm{ZnO}$ nanoneedle synthesis. ${ }^{3}$ The nanostructures obtained were examined by scanning electron microscopy (SEM) using Cambridge 440

\footnotetext{
a)Electronic mail: dalek@ @kusua.hku.hk
}

SEM and LEO 1530 FESEM, x-ray diffractometry (XRD) using a Siemens D5000 x-ray diffractometer, and PL using a $\mathrm{HeCd}$ laser excitation source $(325 \mathrm{~nm})$. Time-resolved PL (TRPL) and time-integrated PL (TIPL) were measured by using the Kerr-gated fluorescence technique. ${ }^{17}$ The experimental apparatus and data analysis methods have been described in detail elsewhere. ${ }^{17}$

Figure 1(a) shows the SEM image of the $\mathrm{ZnO}$ nanoneedles. A higher magnification image of a single needle is given as an inset in Fig. 1(b). It can be observed that the sample consists of thin $(20-60 \mathrm{~nm})$ needles growing out of a $\mathrm{ZnO}$ platelet. The XRD spectrum shows that the fabricated structures are hexagonal $\mathrm{ZnO}$. No peaks due to $\mathrm{Zn}$ or other impurities can be observed. Room-temperature PL spectra excited by a continuous-wave $\mathrm{He}-\mathrm{Cd}$ laser show strong UV emission and very weak orange defect emission, which is different from the commonly reported green defect emission. ${ }^{18}$ The orange emission has been previously reported in $\mathrm{ZnO}$ films. ${ }^{19}$ Unlike the green defect emission which is commonly attributed to an oxygen deficiency, the orange emission has been associated with oxygen-rich $\mathrm{ZnO}{ }^{19}$ The very low intensity of this defect emission indicates that the samples have excellent optical quality.

Figure 2 shows the TIPL spectra of the spontaneous emission and stimulated emission of the $\mathrm{ZnO}$ nanostructures. The spectra have been normalized for easier comparison, since the lasing in the EHP regime has a significantly higher intensity. It can be observed that there is a very small redshift between the spectrum corresponding to the lasing in the E-E scattering regime and the lasing in the EHP regime. The E-E lasing modes are located on the low-energy side of the spontaneous emission, as expected for an E-E scattering process. ${ }^{6,13}$ The threshold for the E-E lasing was $0.48 \mathrm{~mJ} / \mathrm{cm}^{2}$. With increasing pumping power, the spectrum broadens and the intensity becomes significantly higher, which is characteristic of an EHP lasing mode. ${ }^{9,10,13,15}$ The threshold for the EHP emission was $\sim 0.9 \mathrm{~mJ} / \mathrm{cm}^{2}$. However, 

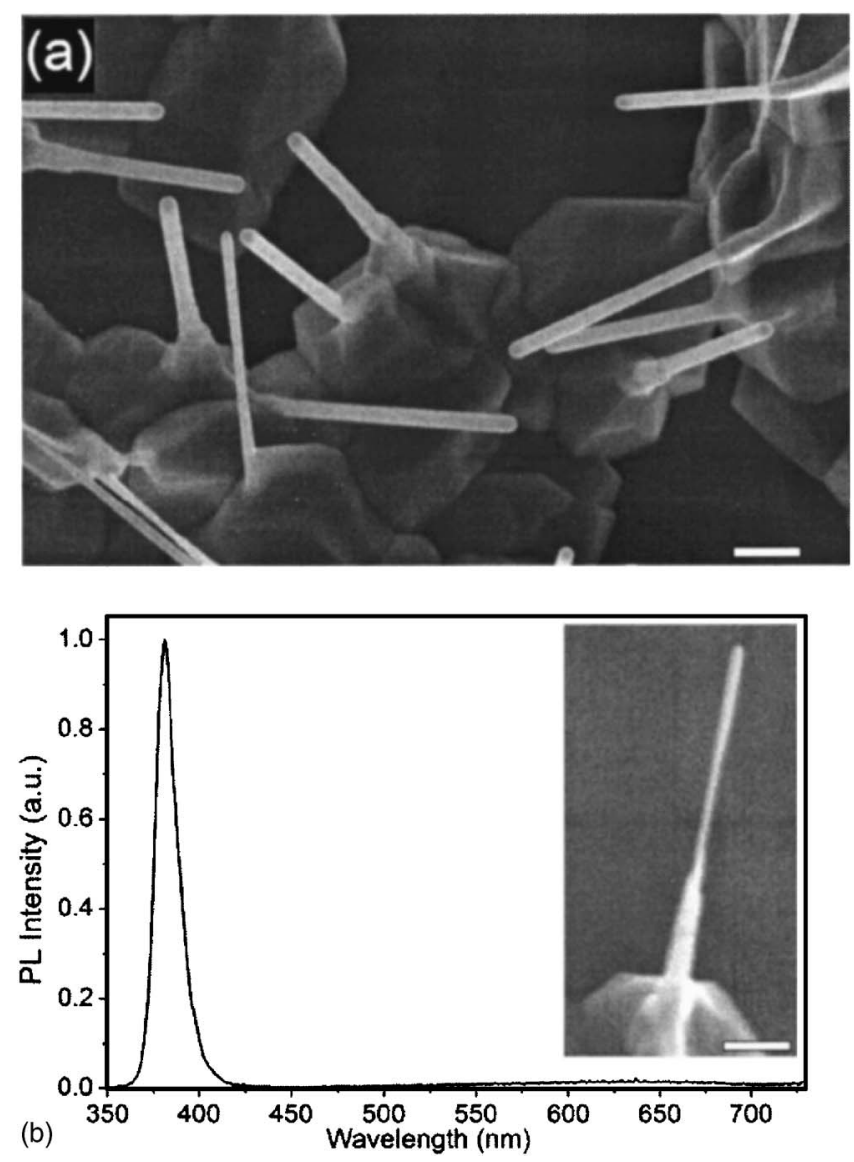

FIG. 1. (a) Representative SEM image of $\mathrm{ZnO}$ nanoneedles. (b) PL spectrum of $\mathrm{ZnO}$ nanoneedles. The inset shows a high magnification SEM image. The scale bar is $200 \mathrm{~nm}$ in both SEM images.

there is no significant redshift between the E-E and the EHP lasing, unlike some previous reports on lasing in $\mathrm{ZnO}$ films. ${ }^{10,13}$

In order to clarify the results obtained here, we performed ultrafast TRPL measurements. These results are shown in Fig. 3. Two observations are noteworthy: (1) The lasing spectrum in the E-E regime shows several different modes, with the width of each mode approximately $\sim 0.7 \mathrm{~nm}$, and (2) there is a small spectral shift of the peaks with time. The modes in the integrated PL spectrum which can be clearly resolved exhibit spacings of $\sim 8 \mathrm{meV}$. The mode spacing in the time-resolved spectra (a detailed analysis was performed for the spectrum at $2 \mathrm{ps}$ ) increases with increasing wavelength from $\sim 0.9 \mathrm{~nm}$ to $\sim 1.4 \mathrm{~nm}$, while the

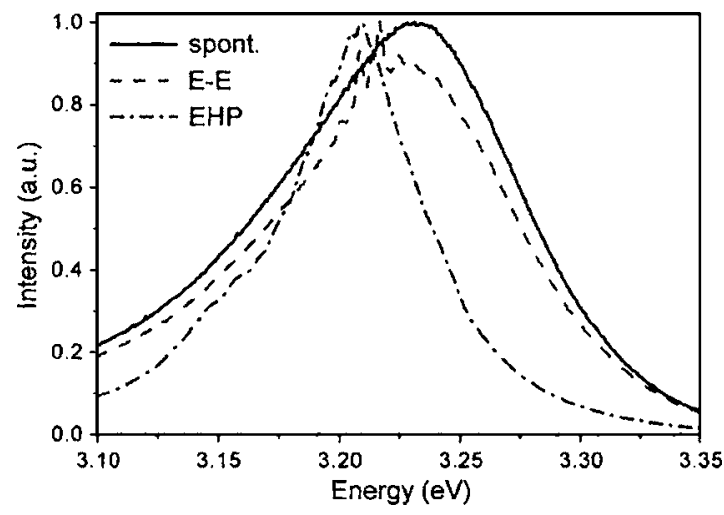
FIG. 2. Time-integrated emission from ZnO nanoneedles. renormalization, it is possible that no new EHP emission line
Downloaded $19 \mathrm{Apr} 2007$ to 147.8 .143 .135 . Redistribution subject to AIP license or copyright, see http://apl.aip.org/apl/copyright.jsp
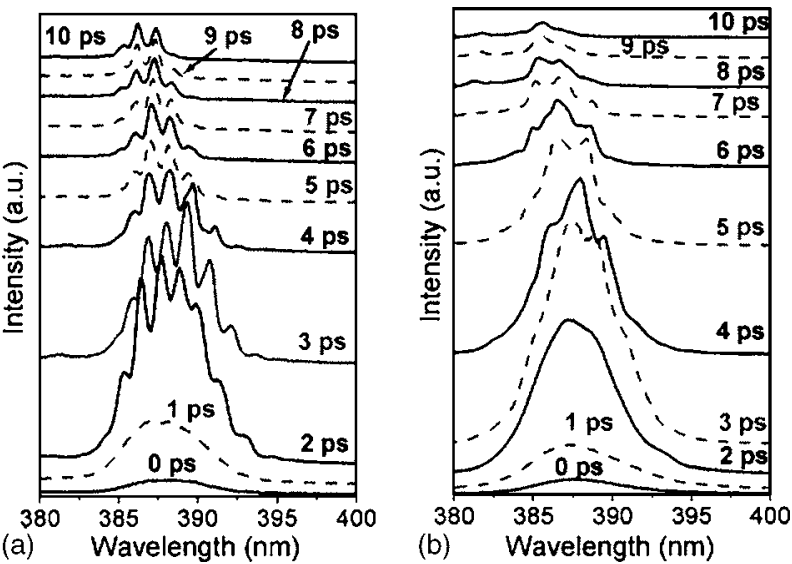

FIG. 3. Time-resolved stimulated emission from $\mathrm{ZnO}$ nanoneedles in (a) E-E scattering regime and (b) EHP regime.

spacing in the energy spacing decreases from $11 \mathrm{meV}$ to 7 $\mathrm{meV}$ with increasing energy. Uneven spacing of the laser modes is likely due to emission originating from different $\mathrm{ZnO}$ nanostructures since there is more than one needle within the laser spot. There are two possible lasing mechanisms in $\mathrm{ZnO}$ nanostructures: Lasing from a cavity formed between two facets of individual $\mathrm{ZnO}$ nanostructures and lasing due to coherent scattering in random media. ${ }^{20}$ If the lasing mechanism is due to coherent scattering, multiple harmonics corresponding to $n D / 2$, where $D$ is the closed loop diameter, should be obvious from the Fourier transform of the lasing spectrum. ${ }^{20}$ However, no periodic peaks appear in the Fourier transforms of TIPL spectrum or TRPL spectra, indicating that the lasing likely originates from different individual nanostructures. It was shown previously that lasing in dendritic nanowire arrays occurs from the ends of the nanorods growing along the [0001] direction. ${ }^{5}$ A similar situation is expected to occur in the $\mathrm{ZnO}$ nanoneedle samples. From the dimensions of the needles and their platelet bases, since the gain is inversely proportional to the cavity length, ${ }^{8}$ it is likely that the lasing occurs along the [0001] direction of the needle growth and that needles provide a dominant contribution to the stimulated emission. In the case of spontaneous emission, however, both needles and platelets contribute to the measured spectra.

For lasing in the EHP mode, a broad peak-with an order of magnitude higher intensity as compared to E-E lasing - can be observed. After $4 \mathrm{ps,} \mathrm{some} \mathrm{structure} \mathrm{in} \mathrm{the}$ peak-at similar energy positions as the E-E lasing modesbegins to emerge. Therefore, the results obtained indicate the coexistence of the EHP and the excitonic recombination. The energy shift between the lasing in the two regimes is very small. The coexistence of the EHP and the E-E scattering in $\mathrm{ZnO}$ films was previously attributed to the spatial nonuniformity of the sample. ${ }^{15}$ The coexistence of the EHP and the E-E scattering was also previously reported for different semiconductor quantum-well and quantum wire structures. $^{21-24}$ The coexistence of excitonic lasing with a spontaneous emission characteristic of EHP has been previously reported for quantum T-shaped wires ${ }^{24}$ and V-shaped wires. ${ }^{23}$ Such a phenomenon would be consistent with the observed very small redshift between the E-E and EHP lasing. It was also shown that due to the competition between the screening of the exciton binding energy and band-gap renormalization, it is possible that no new EHP emission line

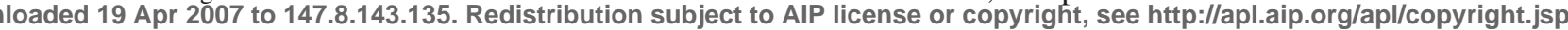




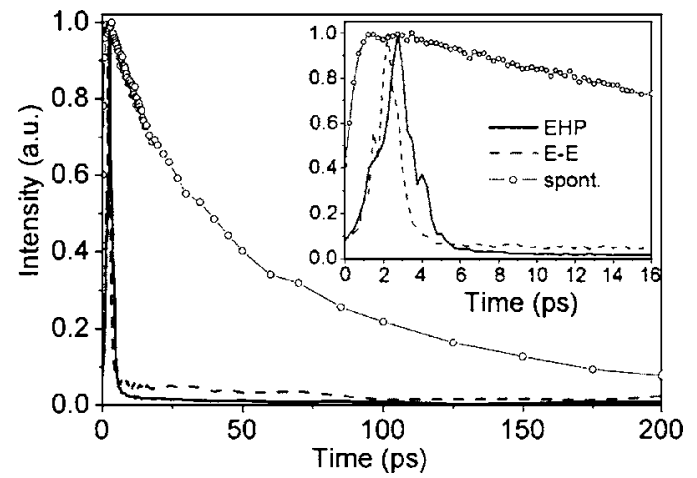

FIG. 4. PL decay curves. The inset shows enlarged region 0-16 ps.

would appear. ${ }^{21,23}$ Localization was shown to significantly affect the lasing mechanism in quantum-well structures. ${ }^{22,23}$ Although our samples have a larger dimension than quantum wire or quantum-well structures (for example, dimensions of a T-shaped nanowire are $14 \mathrm{~nm}$ and $5.6-6 \mathrm{~nm}),{ }^{24}$ it has been shown that the electronic structure of $\mathrm{ZnO}$ nanowires exhibits some size dependent features which differ from bulk $\mathrm{ZnO}^{25}$ Therefore, abrupt changes in the dimension from the $\mathrm{ZnO}$ platelet to a needle may affect the carrier localization due to a higher surface-to-volume ratio of the needles compared to the platelets, and consequently result in different behavior of the stimulated emission compared to lasing in other $\mathrm{ZnO}$ nanostructures reported in the literature.

The decay time of the spontaneous emission and stimulated emission (monitored at $\sim 391 \mathrm{~nm}$ ) is shown in Fig. 4. While the spontaneous emission exhibits biexponential decay with time constants of $20 \mathrm{ps}$ and $87 \mathrm{ps}$, both stimulated emission spectra show very fast decay times of $\sim 1 \mathrm{ps}$. The shortest decay time previously reported for $\mathrm{ZnO}$ lasing is 3 ps. ${ }^{16}$ The rise time of $1 \mathrm{ps}$ for the EHP regime is in agreement with other reports in the literature. ${ }^{9,10,12}$ The decay curve of the stimulated emission in E-E regime shows similar rise time and no significant delay time, unlike $\mathrm{ZnO}$ tetrapod nanostructures ${ }^{12}$ and nanoribbons and nanowires. ${ }^{8}$ Longer delay time until the onset of E-E lasing was previously attributed to the longer time for the weak E-E interaction to produce sufficient scattering events. ${ }^{8}$ The role of the coexistence of EHP and E-E recombination in the absence of the delay time of the lasing in E-E regime needs further study.

To summarize, we fabricated $\mathrm{ZnO}$ nanoneedles and characterized them using TRPL and TIPL. We found that the $\mathrm{ZnO}$ nanoneedles showed very strong UV luminescence and very weak orange defect luminescence. The green defect luminescence was absent in spite of the small size of the nanoneedles. Time-resolved study of the stimulated emission from $\mathrm{ZnO}$ nanoneedles indicated the coexistence of E-E scat- tering and EHP recombination processes, with very fast decay times of the stimulated emission in both E-E and EHP dominated regimes.

The authors would like to thank Amy Wong and Wing Song Lee (Electron Microscopy Unit, HKU) for SEM measurements. This work is partly supported by the Research Grant Council of the Hong Kong Special Administrative Region, China (Project Nos. HKU 1/01C to DLP and HKU 7019/04P to ABD).

${ }^{1}$ S. Y. Li, P. Lin, C. Y. Lee, and T. Y. Tseng, J. Appl. Phys. 95, 3711 (2004). ${ }^{2}$ Q. Wan, K. Yu, T. H. Wang, and C. L. Lin, Appl. Phys. Lett. 83, 2253 (2003).

${ }^{3}$ Y.-K. Tseng, C.-J. Huang, H.-M. Cheng, I.-N. Lin, K.-S. Liu, and I.-C. Chen, Adv. Funct. Mater. 13, 811 (2003).

${ }^{4}$ X. Y. Kong and Z. L. Wang, Nano Lett. 3, 1625 (2003).

${ }^{5}$ H. Yan, R. He, J. Johnson, M. Law, R. J. Saykally, and P. Yang, J. Am. Chem. Soc. 125, 4728 (2003).

${ }^{6}$ Y. Sun, J. B. Ketterson, and G. K. L. Wong, Appl. Phys. Lett. 77, 2322 (2000).

${ }^{7}$ J. C. Johnson, K. P. Knutsen, H. Yan, M. Law, Y. Zhang, P. Yang, and R. J. Saykally, Nano Lett. 4, 197 (2004).

${ }^{8}$ J. C. Johnson, H. Yan, P. Yang, and R. J. Saykally, J. Phys. Chem. B 107, 8816 (2003).

${ }^{9}$ A. Yamamoto, T. Kido, T. Goto, Y. Chen, T. Yao, and A. Kasuya, J. Cryst. Growth 214, 308 (2000).

${ }^{10}$ J. Takeda, S. Kurita, Y. Chen, and T. Yao, Int. J. Mod. Phys. B 15, 3669 (2001).

${ }^{11}$ D. M. Bagnall, Y. F. Chen, M. Y. Shen, Z. Zhu, T. Goto, and T. Yao, J. Cryst. Growth 184, 605 (1998).

${ }^{12}$ Y. H. Leung, W. M. Kwok, A. B. Djurišić, D. L. Phillips, and W. K. Chan, Nanotechnology 16, 579 (2005).

${ }^{13}$ P. Zu, Z. K. Tang, G. K. L. Wong, M. Kawasaki, A. Ohtomo, H. Koinuma, and Y. Segawa, Solid State Commun. 103, 459 (1997).

${ }^{14}$ Z. K. Tang, G. K. L. Wong, P. Yu, M. Kawasaki, A. Ohtomo, H. Koinuma, and Y. Segawa, Appl. Phys. Lett. 72, 3270 (1998).

${ }^{15}$ Ü. Özgür, A. Teke, C. Liu, S.-J. Cho, H. Morkoç, and H. O. Everitt, Appl. Phys. Lett. 84, 3223 (2004).

${ }^{16}$ J. M. Sarko, J. K. Song, C. W. Blackledge, I. Swart, S. R. Leone, S. Li, and Y. Zhao, Chem. Phys. Lett. 404, 171 (2005).

${ }^{17}$ C. Ma, W. M. Kwok, W. S. Chan, P. Zuo, J. T. W. Kan, P. H. Toy, and D. L. Phillips, J. Am. Chem. Soc. 127, 1463 (2005).

${ }^{18}$ A. B. Djurišić, W. C. H. Choy, V. A. L. Roy, Y. H. Leung, C. Y. Kwong, K. W. Cheah, T. K. Gundu Rao, W. K. Chan, H. F. Lui, and C. Surya, Adv. Funct. Mater. 14, 856 (2004).

${ }^{19}$ S. A. Studenikin, N. Golego, and M. Cocivera, J. Appl. Phys. 84, 2287 (1998).

${ }^{20}$ H.-C. Hsu, C.-Y. Wu, and W.-F. Hsieh, J. Appl. Phys. 97, 064315 (2005).

${ }^{21}$ R. Ambigapathy, I. Bar-Joseph, D. Y. Oberli, S. Haacke, M. J. Brasil, F. Reinhardt, E. Kapon, and B. Devaud, Phys. Rev. Lett. 78, 3579 (1997).

${ }^{22}$ P. Chen, J. E. Nichols, M. O. Neill, T. Stirmer, J. H. C. Hogg, B. Lunn, and D. E. Aashenford, J. Appl. Phys. 84, 5621 (1998).

${ }^{23}$ T. Guillet, R. Grousson, V. Voliotis, M. Menant, X. L. Wang, and M. Ogura, Phys. Rev. B 67, 235324 (2003).

${ }^{24}$ J. Rubio, L. Pfeiffer, M. H. Szymanska, A. Pinczuk, S. He, H. U. Baranger, P. B. Littlewood, K. W. West, and B. S. Dennis, Solid State Commun. 120, 423 (2001).

${ }^{25}$ J. Wang, X. An, Q. Li, and R. F. Egerton, Appl. Phys. Lett. 86, 201911 (2005). 